\title{
Lässt sich anhand von klinischen Variablen der Binokularfunktion die langfristige Stabilität der postoperativen Augenstellung nach Korrektur einer intermittierenden Exotropie vorhersagen?
}

\author{
Yidong $\mathrm{Wu}^{\mathrm{a}} \quad$ Meiping $\mathrm{Xu}^{\mathrm{a}} \quad$ Junxiao Zhang ${ }^{\mathrm{a}} \quad$ Jinjing Zhou ${ }^{\mathrm{a}} \quad$ Minghui Wan $^{\mathrm{a}} \quad$ Zhiyue Dai $^{\mathrm{a}}$

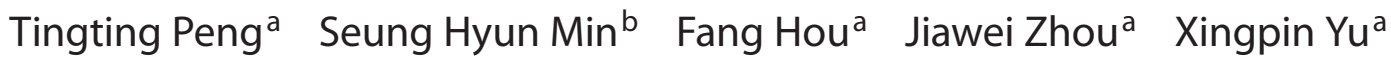 \\ ${ }^{a}$ The Eye Hospital, School of Ophthalmology and Optometry, Wenzhou Medical University, Wenzhou, Zhejiang, China; \\ ${ }^{\mathrm{b}}$ McGill Vision Research, Department of Ophthalmology and Visual Sciences, McGill University, Montreal, QC, Canada
}

\section{Zusammenfassung}

Zielsetzung: Untersuchung, ob klinische Variablen der postoperativen Binokularfunktion die langfristige Stabilität der postoperativen Augenstellung nach Korrektur einer intermittierenden Exotropie bei Kindern vorhersagen können.

Methode: Es erfolgte eine retrospektive Studie zu 39 Kindern (medianes Alter: 7 Jahre), die wegen einer intermittierenden Exotropie operativ behandelt worden waren und bei denen keine Überkorrektur erfolgt war (weniger als 10 Prismen-Dioptrien (PDPT) Exodeviation 1 Monat nach der Operation). Präoperativ sowie 1 Monat und 6 Monate nach der Operation und bei der letzten Nachuntersuchung ( $\geq 24$ Monate) des Patienten wurden die Schielwinkel und Variablen der Binokularfunktion bestimmt. Wir untersuchten die Beziehung zwischen postoperativer Drift (Änderung der Augenstellung) und Variablen der Binokularfunktion (sensorische Funktion, konvergente Fusionsbreite und Stereosehschärfe).

Ergebnisse: Die Erfolgsrate der Operation (Esophorie/-tropie $\leq 5$ PDPT bis Exophorie/-tropie $\leq 10$ PDPT) hatte 6 Monate nach der
Operation auf 76,9\% und zum Zeitpunkt der letzten Nachuntersuchungen der Patienten (im Mittel nach 37 Monaten) auf 53,8\% abgenommen. Die mittlere Exodrift betrug zwischen 1 Monat nach der Operation und der letzten Nachuntersuchung bei Fernfixation $7,7 \pm 9,2$ PDPT $(p<0,001)$. Die Stereosehschärfe in der Ferne, die zentrale Fusion und die konvergente Fusionsbreite verbesserten sich nach der Operation signifikant $(p<0,05)$. Allerdings wurde keine signifikante Korrelation zwischen den Binokularfunktionen zu Beginn jeder Nachbeobachtungsphase und der postoperativen Drift beobachtet (alle $p>0,13$ ).

Schlussfolgerung: Unsere Beobachtungen weisen darauf hin, dass die klinischen Variablen sensorische Fusion, konvergente Fusionsbreite und Stereosehschärfe bei erfolgreich operierten Patienten, die 1 Monat nach der Operation keine Überkorrektur zeigen, nicht als robuste Prädiktoren für die langfristige Stabilität der postoperativen, korrigierten Augenstellung geeignet sind.

(c) 2020 Yidong Wu et al

\section{Einführung}

Die intermittierende Exotropie ist eine Störung, bei der eines der Augen spontan nach außen driftet $[1,2]$. Sie ist die häufigste Exotropie-Form im Kindesalter [3]. In den Vereinigten Staaten sind etwa $1 \%$ der Kinder [3] und in Asien bis zu 3,5\% der Kinder betroffen [4]. Für den Erhalt der Augenstellung bei dieser Form des Schielens wurden kompensatorische fusionale Mechanismen ins Spiel gebracht $[1,5,6]$, die die Ausbildung einer Binokularfunktion erlauben. Ein häufiger Behandlungsansatz bei Patienten, die die Exodeviation (Außenschielen) schlecht kontrollieren können, ist die Schieloperation [1, 2, 7]. Allerdings ist unklar, ob eine Schieloperation den Patienten im Langzeitverlauf hilft, da nach entsprechenden Operationen eine hohe Rezidivrate von intermittierender Exotropie beschrieben wurde [8-11].

In der Vergangenheit wurden bereits mehrere Faktoren untersucht, die mit dem erneuten Auftreten einer intermittierenden Exotropie zusammenhängen könnten, wie das Alter bei Beginn, Refraktionsfehler, die Sehschärfe, der präoperative Schielwinkel, ObliquusDysfunktion, laterale Inkomitanz und frühe Überkorrektur [2, 9, $10,12]$. Allerdings scheint keiner dieser Faktoren Einfluss auf den langfristigen Verlauf nach der Operation zu haben [13]. 
Die Binokularfunktionen (z.B. sensorische Fusion [14], Stereosehschärfe [15] und fusionale Konvergenz [6]) sind wichtige Aspekte bei der Behandlung der intermittierenden Exotropie [1, 2] Sie werden zur Beurteilung des Schweregrads der intermittierenden Exotropie und damit zur Optimierung der Behandlung bestimmt [2]. Darüber hinaus gibt es zunehmende Evidenz, wonach Patienten mit präoperativ besseren Binokularfunktionen (sensorische Fusion, Stereosehschärfe) postoperativ ein besseres sensorisches Ergebnis erzielen könnten [14, 16, 17]. Außerdem lassen die Ergebnisse einer der Studien [14] auf tendenziell schlechtere motorische Ergebnisse (d.h. Rezidiv) bei Patienten mit präoperativer zentraler Suppression schließen. Diese Beobachtungen weisen auf eine prädiktiven Wert der Binokularfunktionen hin. Vor dem Hintergrund dieser früheren Beobachtungen haben wir in dieser Studie die folgende Frage formuliert: Können bessere Binokularfunktionen bei Patienten mit intermittierender Exotropie zu einer stabileren Augenstellung nach einer Operation führen? Unsere Hypothese war, dass die Augenstellung bei Patienten, die nach einer Operation eine bessere Binokularfunktion haben, stabiler ist. Tatsächlich ist man lange davon ausgegangen, dass bei der Ätiologie der intermittierenden Exotropie defekte Fusionsmechanismen eine Rolle spielen $[1,18]$, weshalb die Störung als kortikale Vergenzstörung klassifiziert wurde [19]. Wir mutmaßten, dass Patienten, die nach einer Korrektur der Augenstellung eine schlechte Binokularfunktion aufweisen, sensorische Neuronen verloren haben könnten, die normalerweise die Binokularfunktion unterstützen. Diese Neuronen helfen außerdem, die Augenstellung zu erhalten, indem sie Vergenzreflexe auslösen [20]. Um diese Fragen zu beantworten, entwarfen wir eine retrospektive Studie, in der wir die Beziehung zwischen klinischen Variablen der postoperativen Binokularfunktion und der Stabilität der Augenstellung nach operativer Korrektur untersuchten.

\section{Methode}

Die retrospektive Studie wurde von der Ethikkommission der Universitätsaugenklinik in Wenzhou, China (Eye Hospital, Wenzhou Medical University), genehmigt und im Einklang mit der Deklaration von Helsinki durchgeführt.

\section{Patienten}

Wir unternahmen eine retrospektive Durchsicht der Patientenakten von Kindern unter 16 Jahren, bei denen in der Zeit von Januar 2011 bis Dezember 2014 eine Schieloperation wegen intermittierender Exotropie durchgeführt worden war. Alle Operationen waren von einem der Autoren (XY) vorgenommen worden. Klinische Studien $[9,11,12,21]$ weisen darauf hin, dass eine frühe Überkorrektur innerhalb einer bestimmten Nachbeobachtungsdauer (z.B. 24 Monate) zu einem binokulären Alignment der Augen führen könnte. Deshalb schlossen wir Patienten ein, deren Augen erfolgreich korrigiert worden waren, ohne dass 1 Monat nach der Operation eine Überkorrektur (definiert als 0-10 Prismen-Dioptrien (PDPT) Exodeviation sowohl bei der Fern- als auch bei der Nahfixation) bestand. Die Patienten wurden 6 Monate nach der Ope- ration erneut untersucht und die Nachbeobachtungsdauer betrug mindestens 24 Monate. Patienten mit A-V Pattern, Vertikaldeviation, Obliquus-Dysfunktion und/oder dissoziierter Vertikaldeviation (DVD) schlossen wir aus. Ebenso schlossen wir Patienten mit früherer Schieloperation aus sowie solche, die in der Nachbeobachtungsphase erneut operiert worden waren, und auch Patienten mit Amblyopie (Unterschied zwischen den Augen von $\geq 2$ Zeilen im Snellen-Diagramm), Anisometropie (sphärische oder zylindrische Differenz von $\geq 2$ Dioptrien), neurologischen Anomalien und/oder Entwicklungsverzögerung.

\section{Datenerhebungsmethode}

Aus den Patientenakten wurden präoperative Merkmale wie Geschlecht, bestkorrigierte Sehschärfe und zykloplegische Refraktion sowie das Alter bei der Operation und das Operationsverfahren extrahiert. Außerdem wurden die folgenden Variablen sowohl präoperativ als auch 1 Monat und 6 Monate nach der Operation und bei der letzten Nachuntersuchung des Patienten erhoben: Schielwinkel, Status in Bezug auf die sensorische Fusion, konvergente Fusionsbreite und Stereosehschärfe. Der Schielwinkel in PDPT wurden mit dem alternierenden Prismenabdecktest (PACT) für die Ferne $(6 \mathrm{~m})$ und die Nähe $(1 / 3 \mathrm{~m})$ unter Brillenkorrektur bestimmt. Die sensorische Fusion wurde mit dem 4-Lichter-Test nach Worth in der Ferne und Nähe beurteilt. Die Stereosehschärfe in der Nähe wurde mit dem TNO-Test (Laméris Ootech B.V., Nieuwegein, Niederlande) untersucht, der von 15 bis 480 Winkelsekunden $(\operatorname{arcsec})$ reicht. Die Stereosehschärfe in der Ferne wurde mit einem Optec 3500 Gerät (Stereo Optical Co., Chicago, IL, USA), das von 20 bis 400 arcsec reicht, bestimmt. Bei Patienten, die den Test bei der größten Disparität nicht bestanden, wurde die Stereosehschärfe als «Null» eingestuft. Die fusionale Konvergenz wurde mit einem Synoptophor und Fusionsprüfbildern nachgewiesen [22]. Die Patienten wurden zunächst gebeten, die Röhre so einzustellen, dass ein Summenbild entstand (d.h. Simultansehen). Anschließend wurde die fusionale Konvergenz untersucht, indem die Säulen in diesem korrigierten Winkel festgestellt wurden und die Röhren zueinander hinbewegt wurden (Konvergenz) bis entweder die «Kontrolle» (eine Komponente des Fusionsprüfbildes) verschwand oder das Bild in zwei Teile gespalten wurde (d.h. Aufreißpunkt). Die konvergente Fusionsbreite wurde berechnet, indem der Punkt des Simultansehens vom Aufreißpunkt subtrahiert wurde. Die Messungen mit dem Synoptophor wurden in Grad dokumentiert und in Prismen-Dioptrien umgerechnet. Um eine potentielle Störung der Fusion zu vermeiden, wurde der PACT-Test jeweils am Ende des Untersuchungstermins des Patienten durchgeführt. Darüber hinaus erfolgte, falls erforderlich, zusätzlich ein 1-stündiger Okklusionstest, um ggf. eine präoperative Exotropie vom Pseudodivergenzexzess-Typ zu erkennen [2].

\section{Analyse}

Eine erfolgreiche Operation war definiert als Augenstellung in Primärposition bei Blick in die Ferne mit einer Esophorie/-tropie von $\leq 5$ PDPT bis Exophorie/-tropie von $\leq 10$ PDPT [9]. Die post-
170

Kompass Ophthalmol 2020;6:169-177 DOI: $10.1159 / 000511471$ 
Tab. 1. Demographische und klinische Merkmale von 39 Patienten mit intermittierender Exotropie

\begin{tabular}{|c|c|}
\hline \multicolumn{2}{|l|}{ Merkmal } \\
\hline Geschlecht: weiblich, männlich & $14 ; 25$ \\
\hline Alter bei der Operation: Median (Quartile), Jahre & $7(5 ; 9)$ \\
\hline Präoperative Deviation in der Ferne: Mittelwert \pm SD (95\%-KI), PDPT & $38,8 \times T \pm 8,5(35,9-41,7)$ \\
\hline Präoperative Deviation in der Nähe: Mittelwert \pm SD (95\%-KI), PDPT & $34,4 X T \pm 10,1(31,1-37,7)$ \\
\hline \multicolumn{2}{|l|}{ Exotropie-Typ } \\
\hline Unspezifisch & $29(74,4 \%)$ \\
\hline Konvergenzinsuffizienz & $10(25,6 \%)$ \\
\hline Pseudodivergenzexzess & $0(0 \%)$ \\
\hline Tatsächlicher Divergenzexzess & $0(0 \%)$ \\
\hline \multicolumn{2}{|l|}{ Operationsverfahren } \\
\hline BLR & $8(20,5 \%)$ \\
\hline URR & $28(71,8 \%)$ \\
\hline$B L R+U R$ & $3(7,7 \%)$ \\
\hline
\end{tabular}

operative Drift war definiert als Änderung der Augenstellung vom Untersuchungszeitpunkt 1 Monat nach der Operation bis 6 Monate nach der Operation, bzw. von 1 Monat nach der Operation bis zur letzten Nachbeobachtung oder von 6 Monaten nach der Operation bis zur letzten Nachbeobachtung. Die Vergleiche der Schielwinkel, der Stereosehschärfe und der konvergenten Fusionsbreite (präoperativ, 1 Monat und 6 Monate nach der Operation und bei der letzten Nachuntersuchung) erfolgten mit dem Friedman-Test. Wir führten Posthoc-Analysen mit dem WilcoxonVorzeichen-Rang-Test durch. Der zu den verschiedenen Untersuchungszeitpunkten (präoperativ, 1 Monat und 6 Monate nach der Operation und bei der letzten Nachuntersuchung) festgestellte Status in Bezug auf die sensorische Fusion wurde, einschließlich einer Posthoc-Analyse, mit einem Chi-Quadrat-Test oder einem exakten Test nach Fisher ausgewertet. Die Patienten wurden auf Grundlage ihres Status bezüglich der sensorischen Fusion [14] bei jedem Untersuchungszeitpunkt (präoperativ, 1 Monat und 6 Monate nach der Operation) in zwei Untergruppen eingeteilt: vorhanden (4 Lichter) oder nicht vorhanden (3 oder 2 Lichter). AuBerdem wurden die Patienten auf Grundlage ihrer Stereosehschärfe [17] bei jedem Untersuchungszeitpunkt (präoperativ, 1 Monat und 6 Monate nach der Operation) in zwei andere Untergruppen eingeteilt: hohe Stereosehschärfe ( $\leq 60 \mathrm{arcsec})$ und mittlere bis geringe Stereosehschärfe ( $>60$ arcsec). Für den Vergleich der postoperativen Drift in Untergruppen verwendeten wir den Wilcoxon-Rangsummen-Test. Darüber hinaus untersuchten wir mit dem Korrelationskoeffizienten nach Spearman die Beziehungen zwischen Patientenmerkmalen, sensorischer Funktion, konvergenter Fusionsbreite, Stereosehschärfe und postoperativer Drift. Die Stereosehschärfe wurde bei Analyse als stetige Variable in logarithmische Einheiten transformiert. Patienten mit null Stereosehschärfe wurden der nächsthöheren 0,3-Log-Stufe (d.h. beim TNO-Test 960 arcsec und bei Untersuchung mit dem Optec 3500 Gerät 800 arcsec) zugeteilt [23]. Alle statistischen Analysen erfolgten mit SPSS Version 22.0 (SPSS; Chicago, IL, USA). Hierbei galt ein $p$-Wert von 0,05 als signifikant. Bei multiplen Vergleichen wurde ein $\alpha$-Wert mittels Bonferroni-Korrektur adjustiert.

\section{Ergebnisse}

Insgesamt wurden 39 Fälle eingeschlossen. Das mediane Alter der Patienten bei der Operation betrug 7 (Spannweite: 3-16) Jahre und die mittlere Nachbeobachtungsdauer 37,2 \pm 7,9 (Mittelwert \pm Standardabweichung (SD); Spannweite: 24-52) Monate. Die mittlere Exodeviation betrug präoperativ in der Ferne 34,4 \pm 10 ,1 (95-\%-Konfidenzintervall (95\%-KI): 31,1-37,7) PDPT und in der Nähe 38,8 \pm 9,0 (95\%-KI: 35,9-41,7) PDPT. Tabelle 1 enthält eine Übersicht über die demographischen und klinischen Merkmale der Patienten.

\section{Erfolgsrate der Operation und postoperative Drift}

Die Erfolgsrate betrug 6 Monate nach der Operation 76,9\% und beim letzten Termin 53,8\%. Tabelle 2 zeigt die Erfolgsraten der unterschiedlichen Operationsverfahren.

Der Schielwinkel bei Fernfixation unterschied sich statistisch signifikant $(p<0,001)$ zwischen den verschiedenen Untersuchungszeitpunkten (vor der Operation, 1 Monat und 6 Monate nach der Operation, letzte Nachuntersuchung). Die mittlere Exodrift betrug zwischen 1 Monat nach der Operation und 6 Monaten nach der Operation 3,4 \pm 6,5 (95\%-KI: 1,3-5,5) PDPT ( $p=0,004$; adjustiertes $\alpha=0,0083$ ) und zwischen 1 Monat nach der Operation und letzter Nachuntersuchung 7,7 \pm 9,2 (95\%-KI: 4,7-10,7) PDPT ( $p<$ 0,001 ; adjustiertes $\alpha=0,0083$ ).

Ebenso unterschied sich der in Nahfixation gemessene Schielwinkel statistisch signifikant $(p<0,001)$ zwischen den einzelnen Untersuchungszeitpunkten (vor der Operation, 1 Monat und 6 Monate nach der Operation, letzte Nachuntersuchung). Die mittlere Exodrift betrug zwischen 1 Monat nach der Operation und 6 
Tab. 2. Erfolgsrate unterschiedlicher Operationsverfahren

\begin{tabular}{|c|c|c|c|c|}
\hline \multirow[t]{2}{*}{ Nachbeobachtung } & \multirow[t]{2}{*}{ Methode } & \multirow[t]{2}{*}{ Erfolg (\%) } & \multicolumn{2}{|l|}{ Operationsergebnis } \\
\hline & & & Unterkorrektur (\%) & Überkorrektur (\%) \\
\hline 6 Monate & $\begin{array}{l}\operatorname{BLR}(n=8) \\
\operatorname{URR}(n=28) \\
\operatorname{BLR}+\operatorname{UR}(n=3)\end{array}$ & $\begin{array}{c}6(75) \\
21(75) \\
3(100)\end{array}$ & $\begin{array}{l}2(25) \\
7(25) \\
0(0)\end{array}$ & $\begin{array}{l}0(0) \\
0(0) \\
0(0)\end{array}$ \\
\hline Letzter Termin & $\begin{array}{l}\operatorname{BLR}(n=8) \\
\operatorname{URR}(n=28) \\
\operatorname{BLR}+\operatorname{UR}(n=3)\end{array}$ & $\begin{array}{c}(50) \\
15(53,6) \\
2(66,7)\end{array}$ & $\begin{array}{c}4(50) \\
13(46,4) \\
1(33,3)\end{array}$ & $\begin{array}{l}0(0) \\
0(0) \\
0(0)\end{array}$ \\
\hline
\end{tabular}

BLR = beidseitige Rectus-lateralis-Rücklagerung; URR = einseitige (unilaterale) Rectus-lateralis-Rücklagerung und Rectus-medialisResektion; BLR+UR=beidseitige Rectus-lateralis-Rücklagerung und einseitige Rectus-medialis-Resektion.

Monaten nach der Operation 3,2 $\pm 5,6$ (95\%-KI: 1,4-5,0) PDPT ( $p$ $=0,001$; adjustiertes $\alpha=0,0083)$ und zwischen 1 Monat nach der Operation und letzter Nachuntersuchung 7,9 \pm 9,4 (95\%-KI: 4,8$10,9) \operatorname{PDPT}(p<0,001$; adjustiertes $\alpha=0,0083)$.

\section{Binokularfunktion nach der Operation}

\section{Sensorische Fusion}

Präoperativ zeigten 13 (33,3\%) der 39 Patienten eine normale zentrale Fusion (4 Lichter) in der Ferne. Einen Monat nach der Operation zeigten $30(76,9 \%)$ von 39 Patienten eine normale zentrale Fusion ( $p<0,001$; adjustiertes $\alpha=0,0083$ ). Die Ergebnisse blieben 6 Monate nach der Operation (31 (79,5\%)) und bei der letzten Nachuntersuchung $(31(79,5 \%))$ stabil und unterschieden sich nicht signifikant von dem, was 1 Monat nach der Operation gemessen wurde ( $p=0,784$ bzw. 0,784 ; adjustiertes $\alpha=0,0083$ ). Präoperativ zeigten $29(74,4 \%)$ von 39 Patienten eine normale periphere Fusion (4 Lichter) in der Nähe. Einen Monat nach der Operation zeigten $35(89,7 \%)$ von 39 Patienten eine normale periphere Fusion ( $p=0,138$; adjustiertes $\alpha=0,0083)$. Diese Rate stieg bis 6 Monate nach der Operation leicht auf 37 von 39 an (94,9\%) und blieb bis zur letzten Nachuntersuchung konstant (37 (94,9\%)). Die periphere Fusion unterschied sich 6 Monate nach der Operation und bei der letzten Nachuntersuchung signifikant von der, die 1 Monat nach der Operation beobachtet wurde ( $p=$ 0,025; adjustiertes $\alpha=0,0083$ ).

Fünfzehn $(38,5 \%)$ der Patienten zeigten vor der Operation eine periphere Fusion und zentrale Suppression (d.h. 2 oder 3 Lichter in der Ferne und 4 Lichter in der Nähe). Einen Monat nach der Operation betrug die Anzahl der Fälle 5 (12,8\%), 6 Monate nach der Operation $4(10,3 \%)$ und bei der letzten Nachuntersuchung 6 $(15,4 \%)$

\section{Stereosehschärfe}

Die mediane Stereosehschärfe der Patienten in der Ferne verbesserte sich von Null (Spannweite: 30 arcsec bis Null) vor der Operation auf 70 arcsec (Spannweite: 20 arcsec bis Null) 1 Monat nach der Operation $(p<0,001)$. Die Stereosehschärfe der Patienten in der Ferne war 6 Monate nach der Operation und bei der letzten Nachuntersuchung im Vergleich zum Abschneiden 1 Monat nach der Operation stabil ( $p=0,904$ bzw. 0,928; adjustiertes $\alpha=0,0083$ ). Die mediane Stereosehschärfe der Patienten in der Nähe betrug vor der Operation 60 arcsec (Spannweite: 60 arcsec bis Null), wobei $3(7,7 \%)$ der 39 Patienten eine nicht-messbare Stereosehschärfe hatten. Ihre Stereosehschärfe unterschied sich zu keinem Zeitpunkt nach der Operation signifikant von den Werten vor der Operation ( $p=0,401)$. Siebenundzwanzig von 39 (69,2\%) Patienten hatten bereits vor der Operation eine gute Stereopsis in der Nähe ( $\leq 60$ arcsec) und damit möglicherweise begrenzten Spielraum, ihre Stereosehschärfe in der Nähe nach der Operation weiter zu verbessern. Deshalb unternahmen wir eine zusätzliche Analyse zu Patienten mit mittlerer bis geringer ( $>60$ arcsec) Stereosehschärfe vor der Operation (12 von 39; 30,8\%). Ein Friedman-Test zeigte, dass sich auch bei diesen Patienten die Stereosehschärfe in der Nähe nach der Operation nicht signifikant verändert hatte $(p=0,118)$.

\section{Konvergente Fusionsbreite}

Der Mittelwert der konvergenten Fusionsbreite verbesserte sich von einem Wert von 6,6 $\pm 11,3$ (Spannweite: 0-57,7) PDPT vor der Operation auf 16,6 \pm 13,0 (Spannweite: 0-64,9) PDPT 1 Monat nach der Operation $(p<0,001)$. Die Verbesserung blieb 6 Monate nach der Operation (22,6 $\pm 24,3$; Spannweite: 0-83,9 PDPT) und bei der letzten Nachuntersuchung $(25,0 \pm 26,6$; Spannweite: 0-83,9 PDPT) im Vergleich zum Abschneiden 1 Monat nach der Operation stabil ( $p=0,451$ bzw. 0,179; adjustiertes $\alpha=0,0083$ ).

\section{Beziehung zwischen postoperativer Binokularfunktion und postoperativer Drift}

\section{Postoperative Drift in Untergruppen}

Wir berechneten die Exodrift bei den individuellen Patienten in bestimmten Zeiträumen (d.h. von 1 Monat nach der Operation bis 6 Monate nach der Operation; von 1 Monat nach der Operation bis zur letzten Nachuntersuchung und von 6 Monaten nach der Operation bis zur letzten Nachuntersuchung). Anschließend teil- 
ten wir die Patienten auf der Grundlage ihrer zu Beginn der jeweiligen Nachbeobachtungsphase bestimmten Binokularfunktion (d.h. Fusion und Stereosehschärfe; Einzelheiten siehe Methode) in 2 Untergruppen ein. Patienten, die im 4-Lichter-Test nach Worth 5 Lichter sehen, könnten ein Fusionsvermögen aufweisen. Deshalb schlossen wir diese Patienten aus der Analyse aus (4 Fälle zum Zeitpunkt 1 Monat nach der Operation und 2 Fälle zum Zeitpunkt 6 Monate nach der Operation bei der Bestimmung in der Nähe; 4 Fälle zum Zeitpunkt 1 Monat nach der Operation und 4 Fälle zum Zeitpunkt 6 Monate nach der Operation bei der Bestimmung in der Ferne). Wir analysierten die Differenz der postoperativen Drift in den beiden Untergruppen mit zweiseitigen Wilcoxon-Rangsummentests. Diesen Tests zufolge war die postoperative Drift bei den Patienten mit Fusion (4 Lichter) bzw. ohne Fusion (3 oder 2 Lichter) nicht signifikant unterschiedlich. Ebenso fiel die postoperative Drift bei Patienten mit hoher Stereosehschärfe ( $\leq 60 \mathrm{arcsec}$ ) und solchen mit geringer Stereosehschärfe ( $>60$ arcsec) nicht signifikant unterschiedlich aus. Dies galt für alle Nachbeobachtungstermine und sowohl für die Messungen in der Nähe als auch für die Messungen in der Ferne. Tabelle 3 enthält detaillierte Ergebnisse.

\section{Beziehungen zwischen Stereosehschärfe, Status in Bezug auf die sensorische Fusion, konvergenter Fusionsbreite und post- operativer Drift}

In Abbildung 1 sind die Beziehungen zwischen der zu Beginn jedes Nachbeobachtungszeitraums gemessenen postoperativen Stereosehschärfe und der postoperativen Drift dargestellt. Zwischen der Untersuchung 1 Monat nach der Operation und der letzten Nachuntersuchung wurde nur zwischen einer besseren Stereosehschärfe in der Ferne bei der letzten Nachuntersuchung und einer geringer ausgeprägten postoperativen Drift bei der Nahfixation eine mäßige Assoziation festgestellt ( $\mathrm{rs}=0,484 ; p=$ 0,002). Zwischen der Untersuchung 6 Monate nach der Operation und der letzten Nachuntersuchung wurde eine mäßige Assoziation zwischen einer besseren Stereosehschärfe in der Ferne bei der letzten Nachuntersuchung und einer geringer ausgeprägten postoperativen Drift sowohl in der Ferne ( $r s=0,358 ; p=0,025)$ als auch in der Nähe ( $r s=0,379 ; p=0,017)$ festgestellt. Es wurde keine signifikante Korrelation zwischen der postoperativen Drift und dem Status in Bezug auf die sensorische Funktion oder der konvergenten Fusionsbreite festgestellt.

Die Abbildungen 1(a) und 1(b) zeigen die Beziehung zwischen der 1 Monat nach der Operation gemessenen Stereosehschärfe und der Drift zwischen 1 Monat und 6 Monaten nach der Operation für die Nah- bzw. Fernfixation ( $\mathrm{rs}=-0,17 ; p=0,31 ; \mathrm{rs}=0,21 ; p=$ $0,20)$.

Die Abbildungen 1(c) und 1(d) zeigen die Beziehung zwischen der 1 Monat nach der Operation gemessenen Stereosehschärfe und der Drift zwischen 1 Monat nach der Operation und der letzten Nachuntersuchung für die Nah- bzw. Fernfixation ( $\mathrm{rs}=-0,08$; $p=0,65 ; \mathrm{rs}=0,02 ; p=0,90)$.

Die Abbildungen 1(e) und 1(f) zeigen die Beziehung zwischen der 6 Monate nach der Operation gemessenen Stereosehschärfe und

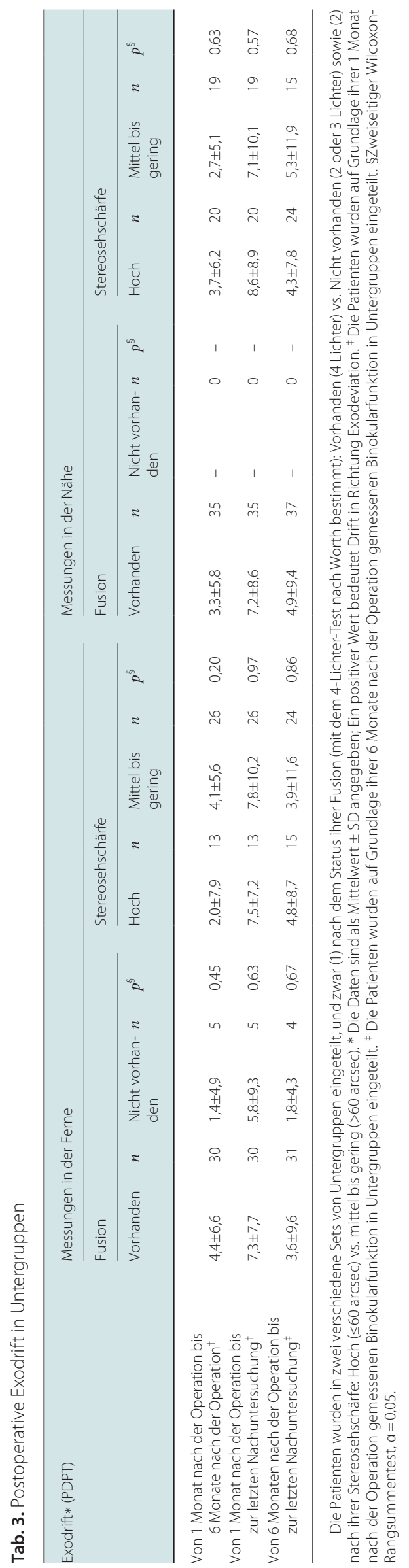




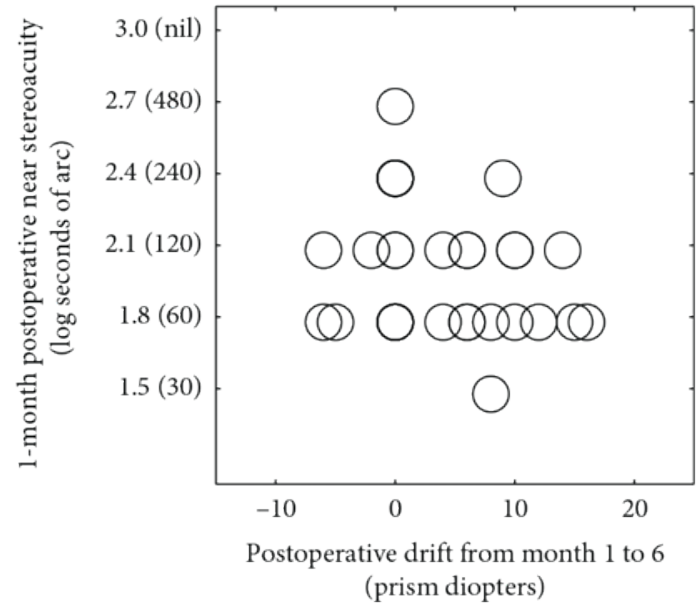

(a)

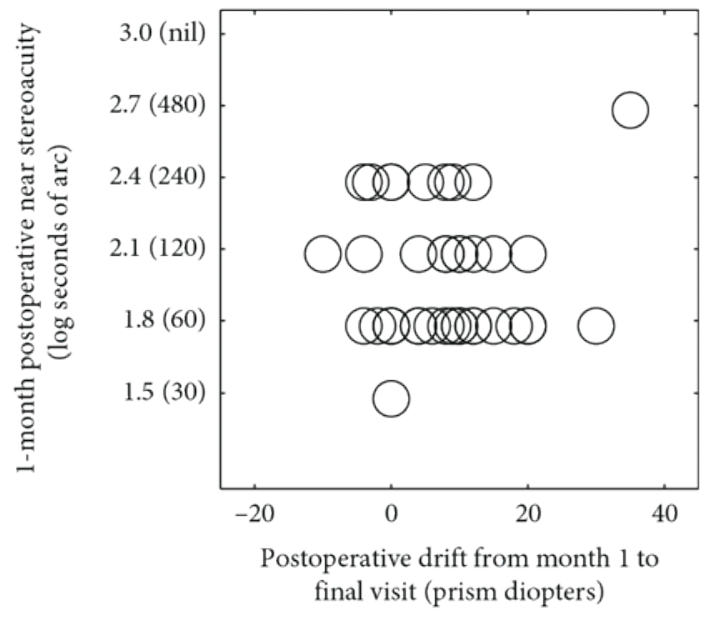

(c)

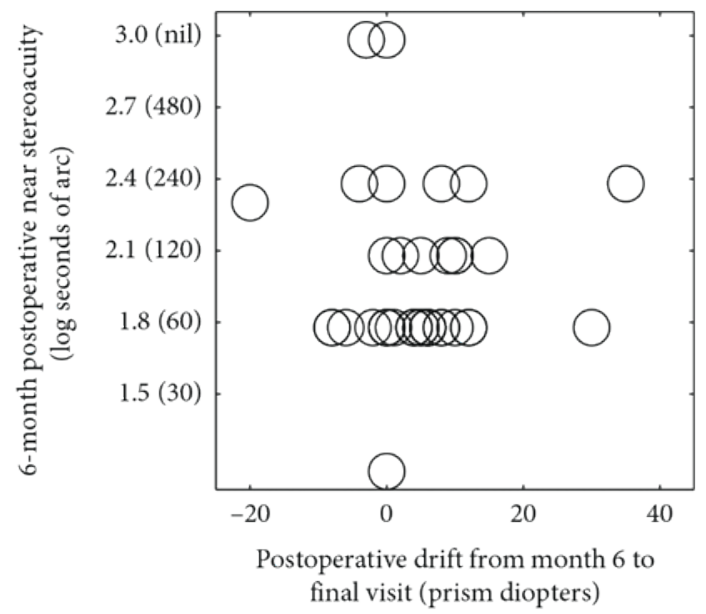

(e)

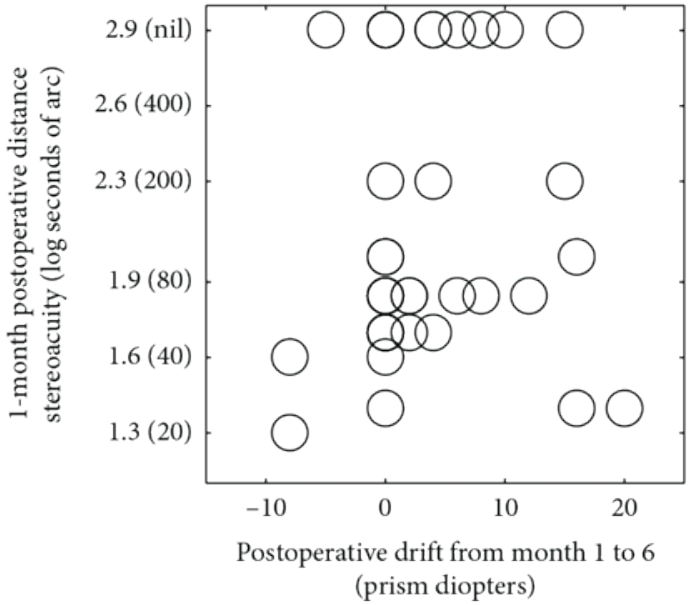

(b)

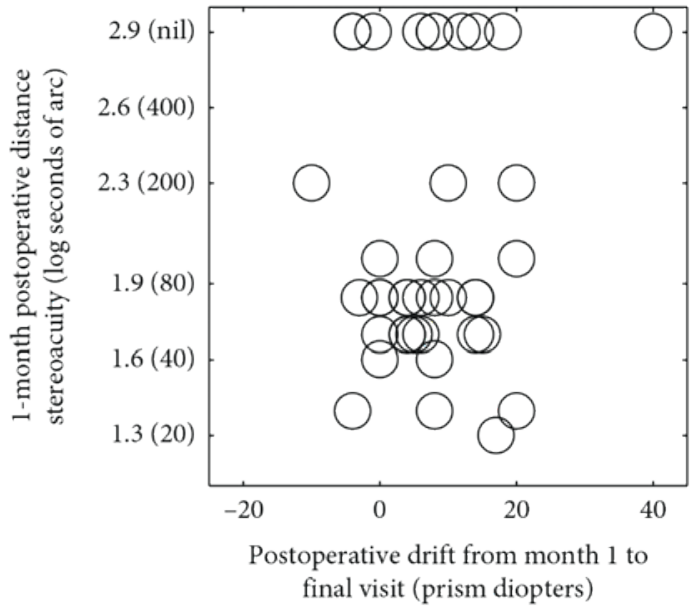

(d)

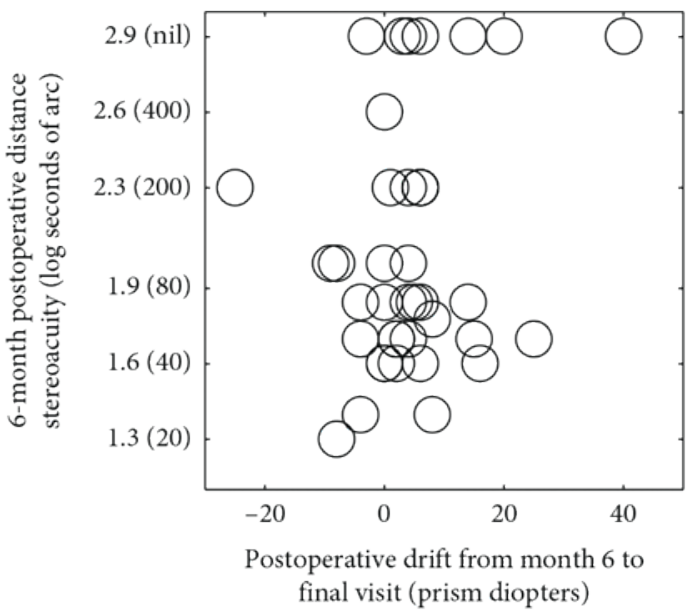

(f)

Abb. 1. Beziehungen zwischen postoperativer Stereosehschärfe und postoperativer Drift in gleicher Entfernung. Alle Diagramme enthalten Daten von 39 Patienten. Die Stereosehschärfe wurde zu Beginn jeder Nachbeobachtungsphase gemessen. Ein positiver Wert zeigt eine Exodrift und ein negativer Wert eine Esodrift an. 
der Drift zwischen 6 Monaten nach der Operation und der letzten Nachuntersuchung für die Nah- bzw. Fernfixation ( $\mathrm{rs}=0,03 ; p=$ $0,85 ; \mathrm{rs}=0,13 ; p=0,45)$.

\section{Zusätzliche Analyse}

Es wurde keine statistisch signifikante (alle $p>0,055$ ) Differenz bei der postoperativen Drift zwischen den verschiedenen Operationsverfahren oder nach Geschlecht bzw. Subtyp der intermittierenden Exotropie beobachtet. Ebenso wurde keine statistisch signifikante Korrelation (alle $p>0,059$ ) zwischen der postoperativen Drift und einem der folgenden präoperativen Merkmale gefunden: Alter bei der Operation, bestkorrigierte Sehschärfe und zykloplegische Refraktion.

\section{Diskussion}

Über die Jahrhunderte haben Augenärzte, Optiker und Neurowissenschaftler die Beziehung zwischen Aspekten der Binokularfunktion und Fehlstellungen der Augen erforscht. In klinischen Untersuchungen zur intermittierenden Exotropie dienten die Variablen der Binokularfunktion als objektive Messwerte für die Beurteilung des Schweregrads der Störung $[14,15,17]$ oder als Beurteilungsvariablen für das sensorische Ergebnis nach einer Operation $[24,25]$. In der vorliegenden Studie untersuchten wir, ob postoperative Variablen der Binokularfunktion als Prädiktor für die langfristige Stabilität der postoperativen Augenstellung nach operativer Korrektur dienen könnten. Wäre dies der Fall, so würde es eine Beziehung zwischen sensorischem und motorischem Behandlungsergebnis geben. Vor der Studie hatten wir die Hypothese aufgestellt, dass eine bessere Binokularfunktion nach der Operation zu einer höheren Stabilität der Augenstellung führen würde. Die Ergebnisse unserer Studie mit einer mittleren Nachbeobachtungsdauer von 37 Monaten widerlegen diese Hypothese. Es gibt wenige Studien, in denen die Beziehung zwischen der postoperativen Binokularfunktion und der Stabilität der postoperativen, korrigierten Augenstellung untersucht wurde. Kushner und Morton [26] weisen darauf hin, dass es bei Erwachsenen mit langfristig bestehendem konstantem Strabismus eine Beziehung zwischen einer Verbesserung der Binokularfunktion nach der Operation (innerhalb von 6 Wochen), die mit Bagolini-Gläsern nachgewiesen wurde, und der langfristigen Stabilität der postoperativen, korrigierten Augenstellung zu geben scheint. Birch und Mitarbeiter [27] zufolge erhöhte eine Stereosehschärfe von Null, die sich unmittelbar nach erfolgreicher Korrektur der Augenstellung (innerhalb von 3 Monaten) entwickelte, bei Kindern mit infantiler Esotropie das Risiko für eine erneute Operation um das 3,6-fache. Zu beachten ist, dass die oben genannten Binokularfunktionen in den beiden Studien in das Muster «mit oder ohne» fallen. Darüber hinaus hatten die untersuchten Patienten (d.h. Kinder mit infantiler Esotropie und Erwachsene mit seit langem bestehendem Strabismus) eine geringere Chance, eine bifoveale Fixation wiederzuerlangen oder zu erzielen, als Patienten mit intermittierender Exotropie [1].
Auf Grundlage unserer Daten erlauben klinische Variablen der postoperativen Binokularfunktion (z.B. sensorische Fusion, Stereosehschärfe und konvergente Fusionsbreite) bei Kindern mit intermittierender Exotropie keine Vorhersage der langfristigen Stabilität einer postoperativen, korrigierten Augenstellung. Eine mögliche Erklärung ist, dass der Schielwinkel [28] und die Stereosehschärfe [23] sowie auch die Kontrolle [29] bei Patienten mit intermittierender Exotropie auch nach der Operation signifikant über den Tagesverlauf schwanken könnten. In neueren prospektiven Studien wurde ein nicht-konsekutives Retest-Verfahren eingesetzt, um die Änderung im Schweregrad der intermittierenden Exotropie zu bestätigen [30, 31]. Dagegen erfolgte bei unseren Patienten nur eine Untersuchung je Nachbeobachtungstermin. Eine weitere mögliche Erklärung könnte sein, dass die hier verwendeten klinischen Verfahren (z.B. 4-Lichter-Test nach Worth für die Fusion; TNO für das stereoskopische Sehen) eher das Ausmaß einer Störung als die Fähigkeit des Patienten messen [20,32]. Entsprechend sind die klinischen Methoden weniger präzise als im Labor angewendete. Beispielsweise beschrieben Holmes et al., dass die meisten Kinder mit intermittierender Exotropie im Vorschul-Randot-Test eine fast normale Stereosehschärfe in der Nähe haben [33]. Dagegen fanden Wu und Mitarbeiter mit einem durch einen Computer generierten Zufallspunkte-Paradigma bei Patienten mit intermittierender Exotropie eine gestörte temporale Integration der Stereopsis [34]. Deswegen könnte der eigentliche Grund darin liegen, dass wir mit den aktuell verwendeten Methoden nicht in der Lage sind, die Störung der Binokularfunktionen nachzuweisen. Demnach muss dieses Problem durch eine zukünftige durchdachte prospektive Studie gelöst werden.

Wir fanden einen mäßigen Zusammenhang zwischen der beim letzten Besuch gemessenen Stereosehschärfe in der Ferne und der Exodrift. Auch wenn diese Ergebnisse keine Vorhersagen erlauben, weisen sie darauf hin, dass Patienten mit besserer Stereosehschärfe in der Ferne eine geringere Exodrift ausbildeten. Demnach weisen unsere Beobachtungen auf eine plausible Hypothese hin, die in zukünftigen Studien untersucht werden könnte: Eine ausgeprägtere Exodrift könnte die Stereosehschärfe in der Ferne stören. Diese Hypothese trifft auf unbehandelte Patienten nicht zu, bei denen Beobachtungen der Pediatric Eye Disease Investigator Group (PEDIG) zufolge ein größerer Schielwinkel nicht mit einer schlechteren Stereosehschärfe verbunden war [35].

Unsere Daten bestätigen die Ergebnisse mehrerer Studien [14, 16, 24, 25]: Die Stereosehschärfe in der Ferne und die zentrale Fusion verbesserten sich bei Patienten mit intermittierender Exotropie nach einer Operation. Auch wenn hier keine statistische Differenz gefunden wurde, wurde bei der Stereosehschärfe in der Nähe und bei der peripheren Fusion nach der Operation ein Trend hin zu einer Verbesserung beobachtet. Darüber hinaus beobachteten wir, dass diese sensorischen Verbesserungen über einen mittleren Nachbeobachtungszeitraum von 37 Monaten nicht signifikant nachließen.

Wir zeigen, dass es einen signifikanten postoperativen Drift in Richtung Exodeviation gab, und zwar über einen kürzeren (von 1 Monat nach der Operation bis 6 Monate nach der Operation) oder 
längeren Zeitraum (von 1 Monat nach der Operation bis zur letzten Nachuntersuchung). Diese Beobachtung steht im Einklang mit der in der Vergangenheit beschriebenen hohen Rezidivrate bei Patienten mit intermittierender Exotropie [8-11]. Die mittlere Exodrift bei der Fernfixation betrug zwischen 1 Monat nach der Operation und letzter Nachuntersuchung (37,2 \pm 7,9 Monate) 7,7 PDPT, was den Werten aus den früheren Studien sehr ähnlich ist. Pukrushpan und Isenberg [36] beschrieben bei Exotropie von 1 Woche bis 36 Monate nach der Operation eine mittlere Exodrift von 8,2 PDPT. Scott und Mitarbeiter [21] fanden bei Exotropie von 1 Woche bis 2 Jahre nach der Operation eine Exodrift in Höhe von 6 bis 11 PDPT, wobei die Gruppe mit intermittierender Exotropie eine etwas ausgeprägtere Drift zeigte.

Die vorliegende Studie weist wie auch frühere Studien [21, 36, 37] darauf hin, dass es bei Patienten mit intermittierender Exotropie zu einer postoperativen Drift kommt. Eine mögliche Erklärung ist, dass der Effekt der Operation mit der Zeit nachlässt. Diese Erklärung wird durch die adaptiven Veränderungen bei den äußeren Augenmuskeln [38] und die aktive Neuroplastizität [39] nach korrigierenden Schieloperationen im Tiermodell unterstützt. Aus einem anderen Blickwinkel könnte man auch die Frage stellen, ob die progressive Exodrift nach einer Operation den natürlichen Verlauf der intermittierenden Exotropie widerspiegelt. Bislang erwies sich der natürliche Verlauf der intermittierenden Exotropie als recht komplex und wurde kontrovers diskutiert. Die herkömmliche Sicht ist die, dass die intermittierende Exotropie eine progressive Erkrankung ist [1]. In einer populationsbasierten Studie fanden Nusz et al. [40], dass die Exodeviation bei mehr als der Hälfte der Patienten über einen Beobachtungszeitraum von 20 Jahren um 10 PDPT oder mehr zunahm, während die Störung bei 3,6\% der Patienten im Beobachtungszeitraum spontan abklang. Die Autoren beschreiben außerdem, dass Patienten, bei denen die Störung über die Zeit abklang, tendenziell geringere Schielwinkel und eine bessere Stereopsis hatten. Dagegen wiesen mehrere Studien darauf hin, dass die intermittierende Exotropie einen stabilen Verlauf hatte und sogar spontan abklang [41, 42]. In jüngerer Vergangenheit unternahm die PEDIG [30] eine randomisierte klinische Studie, der zufolge die ExotropieKontrolle, die Stereosehschärfe und der Grad der Schielstellung bei Kindern mit unbehandelter intermittierender Exotropie über den Beobachtungszeitraum von 3 Jahren stabil waren. Den Daten zufolge scheint die Baseline-Stereosehschärfe keine Vorhersage über die Änderung der Augenstellung zuzulassen. Auf Grundlage der Daten von 89 Patienten mit einer Beobachtungsdauer von 5 Jahren fanden Kwon et al. [37], dass es zu Beginn des 2-JahresZeitraums nach der Operation zu einer Exodrift von fast $90 \%$ $\mathrm{kam}$. Es ist weiterhin unbekannt, ob die progressive Exodrift nach einer Operation eine Regression oder einen natürlichen Verlauf darstellt.

In unserer Studie wählten wir den Zeitpunkt 1 Monat nach der Operation als Baseline für die Beurteilung der postoperativen Exodrift. Dadurch konnten wir potentielle unmittelbare Reaktionen auf und Komplikationen nach der Operation wie Schmerzen, Ödeme, Blutungen und Reaktionen auf die Anästhetika eli- minieren. Darüber hinaus ermöglichte der Zeitraum der Erholung nach der Operation den Patienten, ihre Binokularfunktion wiederherzustellen oder zu entwickeln. Außerdem verwendeten wir relativ strikte Einschlusskriterien, um den Einfluss anderer Faktoren, d.h. Amblyopie, Anisometropie und Vertikaldeviation, möglichst gering zu halten. Daher schlossen wir Patienten aus, die 1 Monat nach der Operation eine Überkorrektur aufwiesen, für die eine Korrelation mit einem langfristigen motorischen Vorteil aber potentiellen Risiko für die Binokularfunktion beschrieben wurde $[9,11,21]$. Allerdings gibt es Aussagen, wonach die schnellste und ausgeprägteste Exodrift im 1. Monat nach der Operation beobachtet wird, vor allem bei Patienten, bei denen 1 Woche nach der Operation eine initiale Überkorrektur vorliegt $[12,43,44]$. Deshalb erhoben wir auch Daten zu den Schielwinkeln der Patienten 1 Woche nach der Operation, um den Einfluss der unmittelbaren postoperativen Phase zu beurteilen. Der Schielwinkel der Exodeviation betrug 0,7 $\pm 2,3$ PDPT (Spannweite: $-4 \sim 10$ PDPT) in der Ferne und 0,6 $\pm 2,2$ PDPT (Spannweite: $-4 \sim 10$ PDPT) in der Nähe. Einer der Patienten hatte 1 Woche nach der Operation eine Esotropie (4 PDPT sowohl in der Ferne als auch in der Nähe). Die mittlere Exodrift betrug zwischen 1 Woche nach der Operation und 1 Monat nach der Operation in der Ferne 1,4 $\pm 3,2$ und in der Nähe 1,1 $\pm 3,1$ PDPT. Diese Ergebnisse weisen darauf hin, dass es weniger wahrscheinlich ist, dass eine initiale Überkorrektur die Hauptfragestellung der Studie verzerrt.

Unsere Studie weist Begrenzungen auf. Erstens kam es durch die retrospektive Datenerhebung zu einem Selektionsbias. Zweitens fehlt in unserer Studie ein Kontrollscore [2], der Auskunft über die Fähigkeit der Patienten gibt, eine Exodeviation zu kontrollieren. Diese Fähigkeit lässt sich nicht direkt aus der Stereosehschärfe oder dem Schielwinkel ableiten [35].

Zusammenfassend lässt sich feststellen, dass die klinischen Variablen sensorische Fusion, konvergente Fusionsbreite und Stereosehschärfe auf Grundlage unserer Ergebnisse nicht als robuste Prädiktoren für die langfristige Stabilität der postoperativen, korrigierten Augenstellung bei Patienten mit intermittierender Exotropie, die erfolgreich operiert wurden und 1 Monat nach der Operation keine Überkorrektur aufweisen, infrage kommen. Wir hoffen, dass unsere Studie Anlass für weitere Studien zum Zusammenhang zwischen Binokularfunktion und Stabilität einer postoperativen, korrigierten Augenstellung geben wird, die neue Erkenntnisse zur intermittierenden Exotropie und eine Orientierung für die klinische Praxis liefern werden.

\section{Danksagungen}

Diese Arbeit wurde durch Fördermittel der Zhejiang Provincial Natural Science Foundation of China (Grant-Nr. LY19H120004) und des Zhejiang Provincial Medical Health Project (Grant-Nr. 2019KY110) an XY unterstützt.

\section{Datenverfügbarkeit}

Die Daten, die den Ergebnissen dieser Studie zugrunde liegen, sind auf Anfrage über den Korrespondenzautor erhältlich.
176

Kompass Ophthalmol 2020;6:169-177 DOI: $10.1159 / 000511471$ 


\section{Offenlegung}

Der Sponsor und die finanzierenden Organisationen waren nicht an Design oder Durchführung dieser Studie beteiligt.

\section{Disclosure Statement}

Die Autoren erklären, dass keine Interessenskonflikte bezüglich der Veröffentlichung dieser Arbeit bestehen.

\section{Lizenzangabe}

Yidong Wu, Meiping Xu, Junxiao Zhang, Jinjing Zhou, Minghui Wan, Zhiyue Dai, Tingting Peng, Seung Hyun Min, Fang Hou, Jiawei Zhou, Xinping Yu: Can Clinical Measures of Postoperative Binocular Function Predict the Long-Term Stability of Postoperative Alignment in Intermittent Exotropia? J Ophthalmol. 2020; 2020: 7392165 (DOI: 10.1155/2020/7392165). ${ }^{\circ}$ 2020 Yidong Wu et al. (Übersetzung), lizensiert unter CC BY 4.0 (https:// creativecommons.org/licenses/by/4.0/deed.de).

\section{Literatur}

Die Literatur ist unter www.karger.com/doi/10.1159/000511471 abrufbar. 